\title{
The characteristics of severe forms of psoriasis on pigmented skins: A retrospective study of 102 cases in Dakar, Senagal
}

\section{Maodo Ndiaye', Fatimata Ly², Pauline Dioussés ${ }^{3}$ Moussa Diallo', Assane Diop², Boubacar Ahy Diatta1, Suzanne Oumou Niang1', Assane Kane', Mame Thierno Dieng ${ }^{1}$}

${ }^{1}$ Department of Dermatology, Hospital Aristide Le Dantec/University Cheikh Anta DIOP Dakar, Dakar, Senagal, ${ }^{2}$ Department of Dermatology, IHS Dakar/University Cheikh Anta DIOP Dakar, Dakar, Senagal, ${ }^{3}$ Department of Dermatology, Hospital Thies/University Thies, Thies, Senagal

Corresponding author: Dr. Maodo Ndiaye, E-mail: maodo7ndiaye@yahoo.fr

\begin{abstract}
Background: Severe forms of psoriasis can be life-threatening for patients. Our objective was to determine the epidemiological, clinical, therapeutic aspects and outcome of severe forms of psoriasis. Methodology: A 18 years retrospective study conducted in the department of Dermatology at Le Dantec hospital listed all the severe forms of psoriasis. Results: We have collected 102 cases of severe forms. The sex ratio was 1.55 and the average age of 47 years. The family histories of psoriasis were reported in 5 patients. Arterial hypertension was noted in 10 patients, diabetes in 4 patients and obesity in 6 patients. Smoking and alcoholism were found respectively in 10 cases and 7 cases. The average duration of the lesions was 2 years. Phytotherapy was reported in 62 patients (62\%). Pruritus was present in 86 patients (84\%). The clinical forms of psoriasis were: erythroderma in 76 cases $(74.5 \%)$, psoriatic arthritis in 14 cases $(13.7 \%)$ and a pustular form in 12 cases (11\%). HIV serology was positive in 12 patients. All patients had received a treatment with topical corticosteroids and emollients. Methotrexate was initiated in 57 patients and retinoids in 27 cases. The outcome was favorable in 67 patients (65\%). We recorded 6 deaths (6\%). Conclusion: The large use of oral phytotherapy medicines and inappropriate treatment precipitated or speeded up the occurrence of severe forms of psoriasis among our patients.
\end{abstract}

Key words: Psoriasis; Severe forms; Black skin; Senegal 


\title{
Les caractéristiques des formes graves de psoriasis sur peaux pigmentées: Étude rétrospective de 102 cas à Dakar, Sénégal
}

\author{
Maodo Ndiaye1, Fatimata Ly², Pauline Dioussé ${ }^{3}$, Moussa Diallo1, Assane Diop², \\ Boubacar Ahy Diatta ${ }^{1}$, Suzanne Oumou Niang' ${ }^{1}$, Assane Kane ${ }^{1}$, Mame Thierno Dieng ${ }^{1}$
}

${ }^{1}$ Department of Dermatology, Hospital Aristide Le Dantec/University Cheikh Anta DIOP Dakar, Dakar, Senagal, ${ }^{2}$ Department of Dermatology, IHS Dakar/University Cheikh Anta DIOP Dakar, Dakar, Senagal, ${ }^{3}$ Department of Dermatology, Hospital Thies/University Thies, Thies, Senagal

Corresponding author: Dr. Maodo Ndiaye, E-mail: maodo7ndiaye@yahoo.fr

\begin{abstract}
RÉSUMÉ
Introduction: Le psoriasis comporte des formes graves qui peuvent engager le pronostic vital. Notre objectif était de déterminer les aspects épidémiologiques, cliniques, thérapeutiques et évolutifs des formes graves de psoriasis. Méthodologie: Une étude rétrospective d'une durée de 18 ans réalisée au service de Dermatologie de l'hôpital le Dantec recensait toutes les formes graves de psoriasis. Résultats: Nous avons colligé 102 cas de formes graves. Le sexratio était de 1.55 et l'âge moyen de 47 ans. Les antécédents de psoriasis familiaux étaient rapportés chez 5 malades. L'hypertension artérielle était notée chez 10 malades, le diabète chez 4 malades et l'obésité chez 6 malades. Le tabagisme et l'éthylisme étaient retrouvés respectivement dans 10 cas et 7 cas. La durée d'évolution moyenne des lésions était de 2 ans. Une phytothérapie était rapportée chez 62 patients (62\%). Le prurit était présent chez 86 malades (84\%). Les formes cliniques de psoriasis étaient; une érythrodermie dans 76 cas (74,5\%), une forme arthropathique dans 14 cas $(13,7 \%)$ et une forme pustuleuse dans 12 cas $(11 \%)$. La sérologie VIH était positive chez 12 malades. Tous les malades avaient reçu un traitement par dermocorticoïdes et émollients. Le méthotrexate était instauré chez 57 malades et les rétinoïdes dans 27 cas. Lévolution était favorable chez 67 malades (65\%). Nous avions enregistré 6 décès (6\%). Conclusion: La large utilisation de la phytothérapie orale et les traitements intempestifs et inappropriés ont précipité à la survenue de formes graves de psoriasis chez nos malades.
\end{abstract}

Mots clés: Psoriasis; Formes graves; Peaux pigmentées; Sénégal

\section{INTRODUCTION}

Considéré comme une maladie inflammatoire généralement bénigne à grand impact psychosocial, le psoriasis comporte certaines formes graves notamment la forme érythrodermique, arthropathique et pustuleuse. Ces dernières peuvent engager le pronostic vital de par leurs complications infectieuses, hydro électrolytiques [1]. En outre, l'association avec le syndrome métabolique et l'infection à VIH peuvent aggraver ce pronostic. En Afrique sub saharienne, les études portant exclusivement sur les formes graves de psoriasis sont rares. Lobjectif de notre étude était de déterminer le profil épidémiologique, clinique, thérapeutique et évolutif des formes graves de psoriasis à la Clinique dermatologique de l'hôpital Aristide Le Dantec.

\section{MATERIALS AND METHODS}

Une étude rétrospective d'une durée de 18 ans (1997-2014) était réalisée au service de Dermatologie de l'hôpital le Dantec de Dakar qui constitue l'un des services de référence en Dermatologie au Sénégal. Les données épidémiologiques, cliniques, thérapeutiques et évolutives ont été recueillies à l'aide d'un questionnaire.

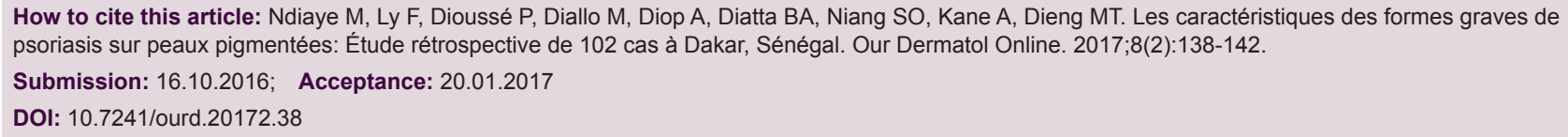


Le diagnostic de psoriasis érythrodermique était basé sur des lésions érythémato-squameuses à squames épaisses avec un érythème vif sur peau pigmentée, confirmé ou non par l'histopathologie cutanée. Pour la forme pustuleuse de Von Zombusch, il s'agit de lésions pustuleuses disséminées disposées en lac avec une confirmation histologique. Enfin pour le psoriasis arthropathique, le diagnostic reposait sur les critères de Vasey et espinoza [2].

Etaient considérées comme psoriasis graves les formes érythrodermique, arthropathique et pustuleuse de Von Zombuch. Etaient exclus tous les malades dont les dossiers sont incomplets. La recherche de comorbidité a été réalisée chez certains patients à savoir un syndrome métabolique et une sérologie rétrovirale. Nous avons inclus tous les malades présentant une forme grave durant cette période d'étude. Les données statistiques étaient saisies et analysées par le logiciel EPI-Info version 6.0. Les tests de chi deux étaient utilisés pour comparer les proportions avec un seuil de significativité de $\mathrm{p}<0,05$.

\section{RESULTS}

Nous avons colligé 120 cas de formes graves mais seuls 102 dossiers étaient exploitables. Le nombre total de psoriasis toutes formes confondues était de 327 cas soit une fréquence de $31 \%$. Tous les malades étaient de phototype VI et ils étaient tous hospitalisés. Ils se répartissaient en 62 hommes pour $40 \mathrm{femmes}$ soit un sex-ratio de 1.55. Lâge moyen était de 47 ans avec des extrêmes de 10 ans et 80 ans. Les antécédents de psoriasis familiaux étaient rapportés chez 5 malades. L'hypertension artérielle était notée chez 10 malades, le diabète chez 4 malades et l'obésité chez 6 malades. Le tabagisme et l'éthylisme étaient retrouvés respectivement dans 10 cas et 7 cas. La pratique de la dépigmentation par les dermocorticoïdes était rapportée chez 10 malades. La durée d'évolution moyenne des lésions était de 2 ans avec des extrêmes de 15 jours à 10 ans. Le psoriasis grave était inaugural chez 10 malades. Une phytothérapie orale et locale à base de plante traditionnelle était rapportée chez 62 patients soit une fréquence de $62 \%$. Les traitements entrepris avant la consultation chez le dermatologue étaient la pénicilline $\mathrm{G}$ retard (Extencilline) dans 20 cas (19\%), la corticothérapie par voie générale dans 15 cas et le cotrimoxazole dans 4 cas. Le prurit était présent chez 86 malades soit une fréquence de $84 \%$ et il existait une association statistiquement significative entre l'existence d'un prurit et la prise de médicaments traditionnels (p: 0,025 odd ratio: 2,55 IC 95\%). Les formes cliniques de psoriasis étaient les suivantes: une érythrodermie (Fig. 1) dans 76 cas (74,5\%), une forme arthropathique (Fig. 2a and b) dans 14 cas $(13,7 \%)$ et une forme pustuleuse (Fig. 3a and b) dans 12 cas (11\%). Quant à la forme arthropathique, l'atteinte articulaire précédait les manifestations dermatologiques dans 1 lcas avec un délai moyen de 3 ans. L'histopathologie cutanée réalisée dans 22 cas, était contributive au diagnostic de psoriasis. A la biologie une élévation des lipides était notée chez 7 malades. Les facteurs rhumatoïdes réalisés chez 6 malades sont négatifs. La sérologie VIH était positive chez 12 malades dont 9 cas de formes érythrodermiques (11\%), 2 cas de formes arthropathique (14\%) et 1 cas de forme pustuleuse.

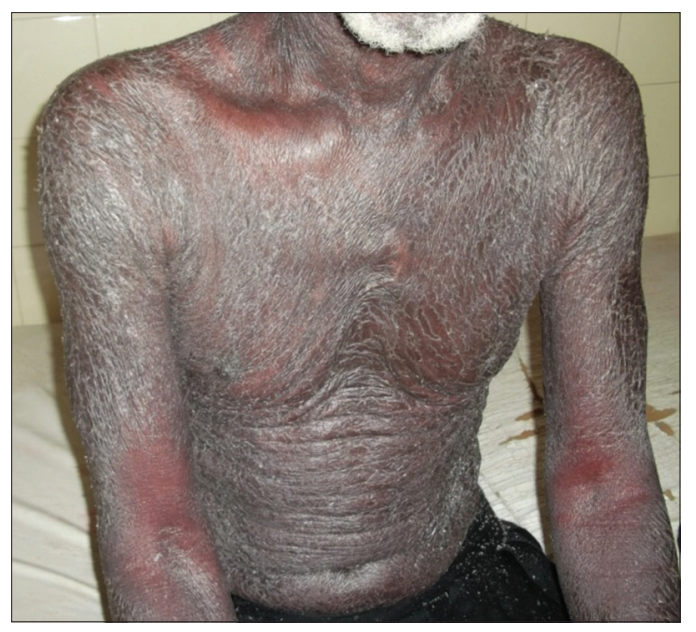

Figure 1: Psoriasis érythrodermique (collection HALD).

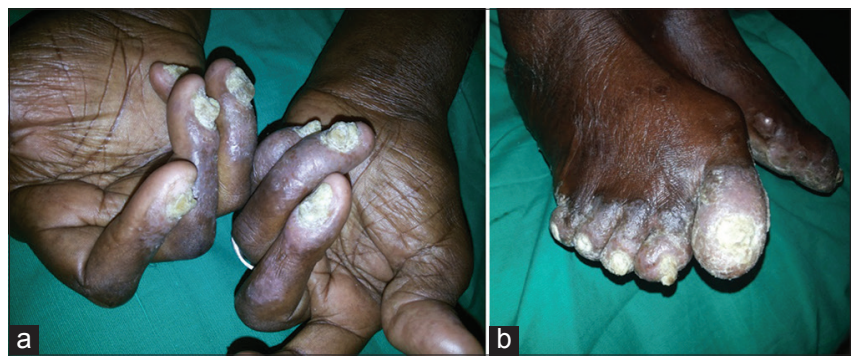

Figure 2: ( $a$ and $b)$ Psoriasis arthropathique (collection HALD).

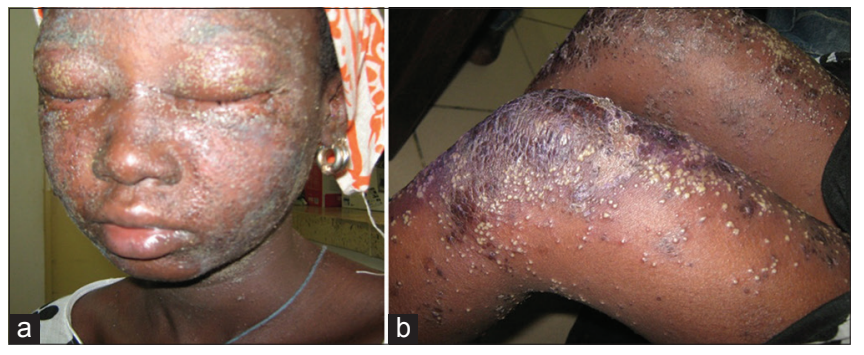

Figure 3: ( $a$ and b) Psoriasis pustuleux (collection HALD). 
Le profil VIH type 1 était prédominant, noté dans 11 cas et tous les malades avaient un taux de CD4 initial inférieur à $350 / \mathrm{mm}^{3}$.

Concernant la forme arthropathique, la radiographie des mains réalisée dans 5 cas, avait objectivé une déminéralisation en bande des métaphyses et un pincement des inters phalangiens distaux chez 3 patients malades. 0n notait des ostéophytes vertébraux chez un malade.

Tous les malades avaient reçu un traitement par dermocorticoïdes et émollients. Les antihistaminiques étaient prescrits chez tous les malades présentant un prurit. Le méthotrexate était institué chez 57 malades $(55 \%)$ et les rétinoïdes dans 27 cas $(26 \%)$. Un traitement antirétroviral était prescrit chez tous les malades présentant une forme associée à l'infection par le VIH. Lévolution était favorable chez 67 malades (65\%) avec un blanchiment quasi complet des lésions après un délai moyen de 8 mois. Une récidive était rapportée chez 12 malades et 27 malades étaient perdus de vue. Nous avions enregistré 6 décès $(6 \%)$ suite aux complications infectieuses.

\section{DISCUSSION}

Cette étude nous a permis de préciser les aspects épidémiologiques, cliniques, thérapeutiques et évolutifs du psoriasis sur peaux pigmentées à la Clinique dermatologique de l'hôpital Aristide Le Dantec. Sur le plan épidémiologique, les formes graves de psoriasis étaient fréquentes puisque représentaient $31 \%$ des psoriasis toute forme confondue. Cette fréquence élevée, pourrait être expliquée d'une part par l'utilisation inappropriée de certaines thérapeutiques telles que la pénicilline retard ou la corticothérapie orale dont ses effets délétères sur le psoriasis sont clairement établis [3]. D'autre part la forte utilisation des plantes médicinales traditionnelles chez nos malades constituerait des facteurs déclenchant ou précipitant l'évolution vers une forme grave de psoriasis [4]. En cas de phytothérapie locale, le mécanisme de frottement de la peau pourrait induire un phénomène de kobner et une aggravation des lésions. Le psoriasis survenait plus sur les adultes relativement jeunes avec une prédominance masculine. Cette dernière était déjà rapportée par une étude antérieurement faite au Maroc par Jilal et al [5]. Une des raisons qui pourrait expliquer cette faible prévalence chez les femmes dans notre contexte, serait l'utilisation de la corticothérapie locale à visée cosmétique qui par son effet favorable sur le psoriasis peut constituer un biais de sélection [6].

Le psoriasis est classiquement une dermatose peu prurigineuse [7]. Cependant chez nos patients; une fréquence accrue prurit était retrouvé dans 84\%. Dans notre contexte, nous avons retrouvé une association statistiquement significative entre l'existence d'un prurit et la prise de médicaments traditionnels (p: 0,025 odd ratio: 2,55 IC 95\%). La présence du prurit pourrait témoigner de l'existence d'une toxidermie associée.

Sur le plan clinique, la forme érythrodermique était dominante puisque représentant les $2 / 3$ de nos malades. D'ailleurs l'étude de Jilal [5] portant sur 160 cas de psoriasis graves, avait rapporté une fréquence élevée de la forme érythrodermique dans $53 \%$. Cette dernière peut poser un problème de diagnostic différentiel avec les autres causes d'érythrodermie mais l'importance des squames épaisses et de l'érythème par son caractère vif sur peau pigmentée oriente le plus vers le diagnostic de psoriasis sans recourir parfois à l'histopathologie cutanée.

Pour le psoriasis arthropathique, sa prévalence dans la population générale n'est pas connue en Afrique subsaharienne [8]. Il se signale habituellement par une atteinte articulaire périphérique, l'atteinte axiale est souvent discrète et il associe très souvent à une rétrovirose [9]. Dans notre série, 14\% des cas psoriasis arthropathiques s'associaient à l'infection par le VIH. En Afrique subsaharienne la plus grande série de rhumatisme psoriasique était rapportée par Njobvu et al. [10] en Zambie; 20 cas (74\%) associés à l'infection par le VIH sur les 27 malades diagnostiqués de psoriasis arthropathique.

Quant à la forme pustuleuse de Von Zombusch, elle est plus rare et que tous les malades avaient eu recours à la phytothérapie orale que nous considérons comme facteur favorisant de l'évolution vers la forme pustuleuse. Elle se discute surtout avec les toxidermies type pustulose exanthématique aigue généralisé.

Par ailleurs, le psoriasis a été la circonstance de découverte de l'infection VIH chez tous nos malades avec immunodépression sévère attestée par un taux de CD4 bas inférieur à 350/ $\mathrm{mm}^{3}$. Peut-on évoquer un lien de causalité en l'immunodépression avancée et la survenue de lésions de psoriasis chez les sujets génétiquement prédisposés ? En tout état de cause, il est licite devant toute forme grave de psoriasis 
survenant de novo chez un adulte de réaliser une sérologie rétrovirale.

Sur le plan thérapeutique les traitements locaux étaient largement utilisés. Le méthotrexate a été le traitement de fond le plus utilisé chez nos malades. Sa large utilisation s'expliquerait par le coût abordable du traitement, sa facilité d'administration, par ses résultats satisfaisants et ses effets secondaires moindres. Les rétinoïdes n'étaient utilisés que dans $26 \%$ de nos malades. Ils étaient prescrits en majorité chez les sujets jeunes présentant des formes rebelles aux traitements locaux dans l'optique d'éviter l'hypofertilité induite par le méthotrexate [11].

Lutilisation des rétinoïdes est limitée dans notre contexte essentiellement par le coût du traitement, de la difficulté d'instituer une contraception et surtout ses impacts quant à l'élévation du bilan lipidique [12] .

Dans notre série, tous les malades associant psoriasis et infection par le VIH étaient blanchis dans un délai moyen de 6 mois après mise en route du traitement antirétroviral. En effet; la diminution de la charge virale induite par le traitement antirétroviral s'accompagne d'une baisse du TNF $\alpha$ dont le rôle est bien établi au cours du psoriasis. En outre l'étude de Kaplan $\mathrm{MH}$ et al montrait que la zidovudine a un effet antiprolifératif [13] donc bénéfique pour la maladie psoriasis.

Lévolution était favorable dans la majorité des cas et les perdus de vue étaient non négligeables. En effet le problème de l'éducation thérapeutique se pose donc avec acuité surtout dans notre contexte de sous médicalisation avec une accessibilité limitée aux thérapeutiques. Souvent négligée, elle est primordiale dans la prise en charge et relève du médecin ou de l'assistante sociale quant à l'information et au soutien du patient dans l'observance du traitement. Ceci permettrait de diminuer le découragement des patients devant le caractère récidivant de la maladie. Les formes graves de psoriasis peuvent engager le pronostic vital comme l'atteste les 6 cas $(6 \%)$ de décès survenus dans un tableau septicémique notés chez nos malades. Une plus faible proportion de 2,5\% était rapportée dans l'étude de Jilal et al [5].

\section{CONCLUSION}

La large utilisation de la phytothérapie orale et les traitements intempestifs et inappropriés ont précipité à la survenue de formes graves de psoriasis chez nos malades. Lérythrodermie rarement inaugurale, était largement majoritaire. Lutilisation de thérapeutique non agressive a permis une évolution favorable dans la majorité des cas.

\section{REFERENCES}

1. Roth P, Grosshans E, Bergoend H. Psoriasis: évolution et complications mortelles. Ann Dermatol Venereol. 1991;118:97-105.

2. Vasey FB, Espinoza LR. Psoriatic arthropathy. In: Calin A (ed). Spondylarthropathies. New York: Grune and Stratton, 1984:151-85.

3. Abel EA, Dicocco LM, Orenberg EK, Fraki JE, Farber EM. Drugs in exacerbation of psoriasis. J Am Acad Dermatol, 1986; 15: 1007-22.

4. Niang SO. Peau et plantes traditionnelles. Progrès en dermatoallergologie, Besançon. 2012, 81-93.

5. Jalal O, Houass S, Laissaoui K, Hocar O, Charioui S, Amal S. Formes graves de psoriasis: à propos de 160 cas. Ann Dermatol Venereol. 2005;132:126-8.

6. Mahe A, Ly F, Aymard G, Dangou M. Skin disease associated with the cosmetic use of bleaching products in women from Dakar, Senegal. Br J Dermatol. 2003;148:493-500.

7. Wallach $\mathrm{D}$, Challier L. Psoriasis de la clinique à la thérapeutique. In John Libbey Eurotext, Paris, 1997:20.

8. Ouedrago D-D, Meyer O. Le rhumatisme psoriasis en Afrique subsaharienne. Rev Rhumat. 2011;78:412-5.

9. Gelfand JM, Gladman DD, Mease PJ, Smith N, Margolis DJ, et al. Epidemiology of psoriatic arthritis in the population of the United States. J Am Acad Dermatol. 2005;53:573.

10. Njobvu P, McGill P. Psoriatic arthritis and immunodeficiency virus infection in Zambia. J Rheumatol. 2000;27:1699-702.

11. Bardaud A. Méthotréxate in psoriasis de la clinique à la thérapeutique. John Libbey Eurotext, Paris, 1997:196.

12. Aubin F. Psoriasis de la clinique à la thérapeutique: Rétinoïde. John Libbey Eurotext, Paris, 1997:196.

13. Kaplan MH, Sadick N, Weider J, Farber B, Neidt G. Antipsoriatic effets of zidovudine in human immunodeficiency virus-associated psoriasis. J Am Acad Dermatol. 2009;20:76-82.

Copyright by Maodo Ndiaye, et al. This is an open-access article distributed under the terms of the Creative Commons Attribution License, which permits unrestricted use, distribution, and reproduction in any medium, provided the original author and source are credited.

Source of Support: Nil, Conflict of Interest: None declared. 